\title{
Entropy estimates for geodesic flows
}

\author{
P. SARNAK \\ Courant Institute for Mathematical Sciences, New York University, 251 Mercer Street, \\ New York, NY 10012, USA
}

(Received 22 August 1981)

Abstract. Let $M$ be a compact Riemannian manifold of (variable) negative curvature. Let $h$ be the topological entropy and $h_{\mu}$ the measure entropy for the geodesic flow on the unit tangent bundle to $M$. Estimates for $h$ and $h_{\mu}$ in terms of the 'geometry' of $M$ are derived. Connections with and applications to other geometric questions are discussed.

\section{Section 1}

Unless otherwise stated, $M$ denotes a compact $\boldsymbol{n}$-dimensional Riemannian manifold of (variable) negative curvature. $S M$ denotes the space of linear elements to $M$ (i.e. the unit tangent bundle), and $\pi: S M \rightarrow M$ the canonical projection. If $v \in S M$ with $\pi(v)=x$, we let $\gamma_{v}(t)$ be the unit speed geodesic starting at $x$ in the direction $v$. The geodesic flow on $S M$ which we denote by $\phi_{t}$ may then be defined by

$$
\phi_{t}(v)=\dot{\gamma}_{v}(t)
$$

If $\mu$ denotes the canonical Riemannian volume element on $S M$, then it is well known that $\mu$ is $\phi_{t}$ invariant. Let $h_{\mu}$ be the entropy of this classical dynamical system $\left\{\phi_{t}, \mu\right\}$ [13] and let $h$ be the topological entropy of the geodesic flow [2]. Quite generally the topological entropy of a dynamical system dominates the measure entropy for any measure, see Goodwyn [5].

$$
h_{\mu} \leq h \text {. }
$$

It is our aim in this paper to give some estimates for $h$ and $h_{\mu}$, in terms of the geometry of $M$, by which we mean, in terms of such quantities as volume, diameter, curvature averages, etc.

It is not too difficult (see Sinai [14]) to show directly that if the curvature $K$ satisfies

then

$$
K_{-} \leq K \leq K_{+}<0
$$

$$
(n-1) \sqrt{-K_{+}} \leq h_{\mu} \leq h \leq(n-1) \sqrt{-K_{-}} .
$$

In dimension 2 Katok [6] has obtained the much sharper isoperemetric type of inequality

$$
h_{\mu} \leq\left(\frac{4 \pi(g-1)}{V}\right)^{1 / 2} \leq h
$$

with strict inequalities unless the curvature is constant (in which case they are all equal). In (1.3) $\mathrm{g}$ is the genus of $M$ while $V$ is the volume. His method uses the fact that every surface is conformal to one of constant curvature, and so in its present form cannot be extended to higher dimensions. 
One is interested in the isometric invariants $h$ and $h_{\mu}$ since they measure the amount of randomness in the geodesic flow. Also $h$ governs the asymptotic behaviour of the lengths of the closed geodesics on $M$, see Margulis [10].

However, we would like to point out how an inequality such as (1.3) can be very useful in some other geometric problems which have received much attention recently. The problem is that of determining the geometry of a manifold from the knowledge of the eigenvalues of the Laplace-Beltrami operator, or 'hearing the shape of a manifold' (see Mckean \& Singer [9]). Katok's inequality (1.3) tells us that the knowledge of the three numbers $h, V$ and $g$ are sufficient to determine whether $M$ is of constant curvature. Now the numbers of $V$ and $g$, may be determined from the asymptotics of the eigenvalues, that is by Weyl's law - see Mckean \& Singer [9]. The lengths of the closed geodesics are also determinable from the eigenvalues, by use of the 'trace formulae' worked out by Chazarin, Colin de Verdiere, Duistermaat Guillemin see for example [4]. As pointed out earlier these lengths (or rather their asymptotics) determine $h$. It follows that one 'can hear' whether a negatively curved surface is of constant curvature or not. Though this result has been proven by other methods, see Berger [1], the above argument is very natural, and inequalities such as (1.3) in higher dimensions could lead to similar results in higher dimensions, where very little on such inverse problems is known.

In order to describe our results we need to define some quantities. Let $v \in S M$ and let $\pi(v)=x$. If $v_{1}, v_{2} \in T_{x}(M)$ are linearly independent, we let $K\left(v_{1}, v_{2}\right)$ be the sectional curvature of $M$ at $x$ in the plane of $v_{1}$ and $v_{2}$. Define the functions $\tilde{K}^{+}$, $\tilde{K}^{-}$on $S M$ by:

and

$$
\tilde{K}^{+}(v)=\max _{\substack{y \in \mathbf{S}_{\mathbf{x}}(M) \\ y \perp v}} K(v, y)
$$

$$
\tilde{K}^{-}(v)=\min _{\substack{y \in S_{\mathbf{x}}(M) \\ y \perp v}} K(v, y)
$$

Let $d \theta$ be the normalized measure on the $(n-1)$ sphere, and define the function $K^{+}$and $K^{-}$on $\boldsymbol{M}$ by

$$
K^{ \pm}(x)=\int_{S_{x}^{n-1}} \hat{K}^{ \pm}(v) d \theta(v)
$$

In terms of these we have:

THEOREM 1.

$$
\frac{n-1}{V} \int_{M} \sqrt{-K^{+}(x)} d V(x) \leq h_{\mu} \leq(n-1)\left(\frac{1}{V} \int_{M}-K^{-}(x) d V(x)\right)^{1 / 2}
$$

where $d V$ is the Riemannian volume element.

Firstly we note that in constant curvature all of the above numbers are the same. Secondly, the above clearly is significantly sharper than (1.2). Thirdly, in dimension two where $K^{+}(x)$ and $K^{-}(x)$ are just the Gauss curvature $K(x)$, the above leads 
through the use of the Gauss-Bonnet formula to:

CROROLlaRY 1. For a surface of genus $g \geq 2$

$$
\frac{1}{V} \int_{M} \sqrt{-K(x)} d V(x) \leq h_{\mu} \leq\left(\frac{4 \pi(g-1)}{V}\right)^{1 / 2} .
$$

The right hand side in this corollary is a slightly veaker version of (1.3).

The proof of theorem 1 also allows one to bound $h_{\mu}$ from above, even for spaces with positive curvature, as long as the curvature is not too large. We state and prove the following theorem only in the case of dimension two (the $n$-dimensional case is similar).

THEOREM 2. Let $M$ be a surface of genus $g \geq 2$ and suppose that the curvature (which may be positive) satisfies

$$
K(x) \leq \frac{4 \pi(g-1)}{V}
$$

then

$$
h_{\mu} \leq 2\left(\frac{4 \pi(g-1)}{V}\right)^{1 / 2} .
$$

Before giving proofs, which will all be carried out in $\$ 2$, we discuss some lower bounds for the topological entropy $h$. The lower bound is in terms of the spectrum of the Laplace-Beltrami operator on the universal covering. One may argue that this spectrum is no easier to compute, however, we are following the principle that eigenvalues are 'more computable' than entropy, just as in the case of a diffeomorphism on a compact manifold, where a lower bound for $h$ is provided in terms of eigenvalues of the induced map on real homology, see Bowen [2]. As pointed out in Manning [7], in the case of a flow, $\phi_{1}$ is isotopic to the identity so that these mentioned bounds lead to nothing.

Let $M$ be the universal covering of $\tilde{M}$, thus $M$ is diffeomorphic to $\mathbb{R}^{n}$, and is locally isometric with $\dot{M}$. Let $\tilde{\Delta}$ be the corresponding Laplace-Beltrami operator on functions on $\dot{M}$. Let $\beta$ be the bottom of the spectrum of $-\tilde{\Delta}$ with respect to the $L^{2}$ spectral theory of $\tilde{\Delta}\left(L^{2}\right.$ with respect to the Riemannian volume measure of $\tilde{M})$. We cannot describe $\beta$ as the smallest eigenvalue of $-\tilde{\Delta}$, since $\tilde{M}$ is not compact, and so square integrable eigenfunctions should not be expected. However, $\beta$ may be described as the largest real number $\gamma$ such that all numbers to the left of are in the resolvent set of $-\tilde{\Delta}$.

THEOREM 3. Notation as above then, the spectrum of $-\tilde{\Delta}$ is essential and $\beta \leq h^{2} / 4$.

We shall see that the above is sharp for constant curvature spaces. Also, the left-hand side is greater than or equal to $\left((n-1)^{2} / 4\right)\left|K_{+}\right|$by a result which is due to Mckean [8]. Thus the estimate of theorem 3 is no worse than (1.2), though in many cases the lower bound for $h$ via

$$
h \geq h_{\mu} \geq \frac{n-1}{V} \int_{M} \sqrt{-K^{+}(x)} d V(x)
$$


from (1.1) and theorem 1, may be better. We also construct at the end of $\S 2$ a sequence of surfaces for which $h \rightarrow \infty$ but for which $\beta$ is bounded above, so that the estimate of theorem 3 may be very poor indeed. Finally we remark that the inequality of theorem 3 may of course be viewed as an upper bound for $\beta$.

\section{Section 2}

All expressions for entropy involve quantities defined through limits of one sort or another. We choose appropriate expressions from which such estimates can be drawn. In the case of the measure entropy $h_{\mu}$, we will use a formula due to Pesin [11] which is in terms of Liapunov exponents. For the topological entropy $h$ we will use a characterization due to Margulis [10] and Manning [7] in terms of growth rates of geodesic balls in $M$.

We begin with Pesin's formula. We need an appropriate Riemannian structure on $S M$. Let

$$
K: T(T N) \rightarrow T M
$$

be the connection map (see Eberlein [3]). The tangent space to $T M$ then splits into a sum of a horizontal and vertical subspace

$$
(T M)_{v}=\operatorname{ker} d \pi \oplus \operatorname{ker} K, \quad \text { where } v \in T M .
$$

A Riemannian structure on $T M$ is then given by

$$
\langle\xi, \eta\rangle=\langle d \pi \xi, d \pi \eta\rangle_{\pi v}+\langle K \xi, K \eta\rangle_{\pi v}
$$

for $\xi, \eta \in T_{v}(T M) . S M$ then inherits the induced Riemannian structure as a subspace of $T M$. The measure $\mu$ is just the volume element for this structure.

Now fix a $v \in S M$ and $\xi \in T_{v}(S M)$, and let \|\| be the norm on $T(T M)$ just described. We define the Liapunov exponent at $v$ in the direction $\xi$ by

$$
\chi^{+}(v, \xi)=\varlimsup_{t \rightarrow \infty} \frac{1}{t} \log \left\|d \phi_{t}(\xi)\right\| .
$$

It is clear that $\chi^{+}$assumes at most $2 n-1$ distinct values on $T(S M)$, and we have a filtration

$$
L_{0}(v) \underset{\neq}{\subset} L_{1}(v) \subset \cdots \underset{\neq}{\subset} L_{i(v)}=T_{v}(S M)
$$

where $\chi^{+}(v, \xi)$ is constant at value $\lambda_{j}(v)>0$ on $L_{j}(v) \backslash L_{j-1}(v), j \geq 1$, and $\chi^{+}(v, \xi) \leq 0$ for $\xi \in L_{0}(v)$. The $\lambda_{j}$ 's are distinct and increasing in size. Let

Define

$$
k_{j}(v)=\operatorname{dim} L_{j}(v)-\operatorname{dim} L_{j-1}(v) \text { for } j \geq 1 \text {. }
$$

$$
\chi(v)=\sum_{j=1}^{i(v)} k_{j}(v) \lambda_{j}(v)
$$

Pesin's formula for $h_{\mu}$, which applies whenever $\mu$ is a smooth measure for a flow, states that

$$
h_{\mu}=\int_{S M} \chi(v) d \mu(v)
$$

In order to estimate the exponents $\chi(v)$ we need a more concrete form for the differential $d \phi_{t}(\xi)$. This can be done by use of Jacobi fields along the geodesic $\gamma_{v}(t)$. 
A Jacobi field along $\gamma$ is a vector field $Y$ which satisfies

$$
Y^{\prime \prime}+R_{x y} X=0
$$

where $X=\dot{\gamma}(t)$, ' is covariant differentiation along $\gamma$, and $R$ is the curvature tensor. An isomorphism between $T_{v}(T M)$ and the Jacobi fields on $\gamma$ comes from solving (2.4) with

$$
Y(0)=d \pi(\xi), \quad Y^{\prime}(0)=K(\xi) .
$$

This correspondence has the added property that

$$
\left\|d \phi_{t}(\xi)\right\|^{2}=\left\|Y_{\xi}(t)\right\|^{2}+\left\|Y_{\xi}^{\prime}(t)\right\|^{2} .
$$

For a proof and more details see Eberlein [3].

Since we are interested in the exponential growth rate of $d \phi_{t}(\xi)$ we may restrict ourselves to perpendicular Jacobi fields, i.e. ones for which $\left\langle Y_{\xi}(t), \dot{\gamma}(t)\right\rangle=0$ for all $t$. To analyse these it is convenient to choose a parallel orthonormal frame $E_{1}, \ldots, E_{n}$ along $\gamma$ with $E_{n}(s)=\dot{\gamma}(s)$ (here $s=t=$ arclength parameter). A perpendicular field $Y(s)$ may then be expressed as

$$
Y(s)=\sum_{i=1}^{n-1} Y_{i}(s) E_{i}(s)
$$

If $R(s)$ is the $(n-1) \times(n-1)$ matrix with entries

$$
\boldsymbol{R}_{i j}(s)=\left\langle\boldsymbol{R}_{E_{n}(s) E_{i}(s)} E_{n}(s), E_{j}(s)\right\rangle,
$$

then (2.4) becomes the vector equation

$$
Y=\left(\begin{array}{c}
Y_{1} \\
\vdots \\
Y_{n-1}
\end{array}\right), \quad \frac{d^{2} Y}{d s^{2}}=-R(s) Y(s)
$$

Of course, $R_{i j}$ is symmetric and $Y^{T} R Y /\|Y\|^{2}$ is just the sectional curvature in the plane of $Y(s)$ and $\dot{\gamma}(s)$. It follows that the spectrum of the matrix $R(s)$ lies in the interval

$$
\left[\hat{\boldsymbol{K}}^{-}(\dot{\gamma}(s)), \hat{\boldsymbol{K}}^{+}(\dot{\gamma}(s))\right]
$$

LEMMA 2.1. Let

$$
g(t)=\|Y(t)\|^{2}+\left\|Y^{\prime}(t)\right\|^{2}
$$

then if for some initial conditions $\left(Y(0), Y^{\prime}(0)\right)$ it is true that

$$
\int_{0}^{\infty} g(u) d u=\infty
$$

then

$$
\varliminf_{t \rightarrow \infty} \frac{\log g(t)}{t} \geq \varliminf_{t \rightarrow \infty} \frac{2}{t} \int_{0}^{t} \sqrt{-\hat{K}^{+}(s)} d s .
$$

Proof. let $Z(t)=\langle Y(t), \dot{Y}(t)\rangle$. Notice that the $\mathbb{R}^{n-1}$ inner product of

$$
Y=\left(\begin{array}{c}
Y_{n} \\
\vdots \\
Y_{n-1}
\end{array}\right)
$$


and that of (2.6) in $T_{\gamma(s)}(M)$ are the same.

$$
\frac{d z}{d t}=\langle\dot{Y}, \dot{Y}\rangle+\langle Y,-R Y\rangle \geq\|\dot{Y}\|^{2}+\left(-\hat{K}^{+}\right)\|Y\|^{2} .
$$

Now

$$
\begin{aligned}
\|\dot{Y}\|^{2}+\left(-\hat{K}^{+}\|Y\|^{2}\right. & \geq 2 \sqrt{-K^{+}}\|Y\|\|\dot{Y}\| \\
& \geq 2 \sqrt{-K^{+}}(t)\langle Y, Y\rangle \\
& =2 \sqrt{-K^{+}(t)} z(t) \\
\frac{d z}{d t} & \geq 2 \sqrt{-K^{+}(t)} z(t) .
\end{aligned}
$$

Now by compactness there is a $0<c<1$ such that $-K^{+}(t) \geq c$, and so by (2.9)

$$
\frac{d z}{d t} \geq c g(t)
$$

The assumption that $\int_{0}^{\infty} g(u) d u=\infty$, then shows that $z\left(t_{0}\right)>0$ for some $t_{0}$. Now use (2.10) to conclude that

$$
\frac{\log z(t)}{t} \geq \frac{2}{t} \int_{t_{0}}^{t} \sqrt{-K^{+}(u)} d u
$$

and since $g(t) \geq 2 z(t)$, the conclusion of lemma 2.1 is clear.

Lemma 2.1. Again let

$$
g(t)=\|Y(t)\|^{2}+\left\|Y^{\prime}(t)\right\|^{2},
$$

with $Y$, as in (2.6) and satisfying (2.7). Let $c>0$ be arbitrary, then

$$
\varlimsup_{t \rightarrow \infty} \frac{\log g(t)}{t} \leq c+c^{-1} \varlimsup_{t \rightarrow \infty} \frac{1}{t} \int_{0}^{t}\left(-\hat{K}^{-}\right)(u) d u .
$$

Proof. Let

$$
\begin{aligned}
z(t) & =c\|Y\|^{2}+c^{-1}\left\|Y^{\prime}\right\|^{2} \\
\frac{d z}{d t} & =2 c\langle Y, \dot{Y}\rangle+2 c^{-1}\langle\dot{Y},-R Y\rangle \\
& =Y^{T}\left(2 c-2 c^{-1} R\right) Y \\
& \leq 2\|\dot{Y}\|\|Y\|\left\|c+c^{-1}(-R)\right\|
\end{aligned}
$$

(the last \|\| being $L^{2}$ matrix norm). Now

Therefore

$$
\left\|c+c^{-1}(-R)\right\| \leq c+c^{-1}\left(-\hat{K}^{-}\right) .
$$

$$
\frac{d z}{d t} \leq\left(c+c^{-1}\left(-\hat{K}^{-}\right)\right) z
$$

and so

$$
\varlimsup_{t \rightarrow \infty} \frac{\log z(t)}{t} \leq c+c^{-1} \overline{\lim } \frac{1}{t} \int_{0}^{t}\left(-\hat{K}^{-}\right)(u) d u .
$$

Finally, we have $g(t) \leq B z(t)$ for a suitable constant $B$, from which the lemma follows. 
If we choose

$$
c=\sqrt{\varlimsup_{t} \frac{1}{t} \int_{0}^{t}-\hat{K}-(u) d u}
$$

in the last lemma we have

$$
\varlimsup_{t \rightarrow \infty} \frac{\log g(t)}{t} \leq 2\left(\varlimsup_{t \rightarrow \infty} \frac{1}{t} \int_{0}^{t}-K^{-}(u) d u\right)^{1 / 2} .
$$

We now prove theorem 1 . Since all the sectional curvatures are negative the flow $\phi_{t}$ is Anosov on $S M$. Thus there is a decomposition of $T_{v}(S M)$ as

$$
T_{v}(S M)=X \oplus Y \oplus Z
$$

with $\operatorname{dim} X=\operatorname{dim} Y=n-1, \operatorname{dim} Z=1 . X$ is the tangent space to the contracting horospheres, $Y$ to the expanding horospheres, and $Z$ is the flow direction. For suitable $\lambda>0$ we have

$$
\begin{aligned}
\left\|d \phi_{t}(\xi)\right\| \leq \mathrm{e}^{-\lambda t}\|\xi\|, & t>0, \xi \in X \\
\left\|d \phi_{-t}(\xi)\right\| \leq \mathrm{e}^{-\lambda t}\|\xi\|, & t>0, \xi \in Y
\end{aligned}
$$

and $\dot{\gamma}(0)=Z$.

From this it is clear that $\chi(v, \xi) \leq 0$ for

$$
\xi \in X \oplus Z \text {. }
$$

On the other hand, for any $\xi$ we have seen that (by (2.13))

$$
\chi(v, \xi) \leq \varlimsup_{t \rightarrow \infty}\left(\frac{1}{t} \int_{0}^{t}-\hat{K}^{-}(u) d u\right)^{1 / 2}
$$

(since $\left\|d \phi_{t}(\xi)\right\|^{2}=g(t)$ ).

(2.14) and definition (2.3) show that

$$
\chi(v) \leq(n-1)\left(\varlimsup_{t \rightarrow \infty} \frac{1}{t} \int_{0}^{t}-\hat{K}^{-}(u) d u\right) .
$$

For a lower bound we observe the easily verified fact that for $\xi \notin Z \oplus X_{v}$

$$
\left\|d \phi_{t}(\xi)\right\| \rightarrow \infty \quad \text { as } t \rightarrow \infty .
$$

Therefore

$$
g(t)=\left\|Y_{\xi}(t)\right\|^{2}+\left\|Y_{\xi}^{\prime}(t)\right\|^{2} \rightarrow \infty
$$

as $t \rightarrow \infty$. (Note that $\xi \in X_{v}$ or $y_{v} \Rightarrow Y_{\xi}$ is perpendicular Jacobi field.)

Applying (2.8) gives

$$
\chi(v, \xi) \geq \varliminf_{t \rightarrow \infty} \frac{1}{t} \int_{0}^{t} \sqrt{-\hat{K}^{+}(u)} d u .
$$

The dimension of the space on which the latter holds is $(n-1)$ dimensional, therefore

$$
\chi(v) \geq(n-1) \varliminf_{t \rightarrow \infty} \frac{1}{t} \int_{0}^{t} \sqrt{-\hat{K}^{+}(u)} d u
$$


520

P. Sarnak

By the Birkhoff ergodic theorem

and

$$
\lim _{t \rightarrow \infty} \frac{1}{t} \int_{0}^{t} \sqrt{-\hat{K}^{+}(u)} d u=\psi_{1}(v)
$$

$$
\lim _{t \rightarrow \infty} \frac{1}{t} \int_{0}^{t}-\hat{K}^{-}(u) d u=\psi_{2}(v)
$$

exists for almost all $v$, and the integral of the limit (which in this case is constant almost everywhere) is simply the space average. Therefore

$$
\begin{aligned}
(n-1) \int_{S M} \sqrt{-\hat{K}^{+}(v)} d \mu_{1}(v) & \leq h_{\mu}=\int_{S M} \chi(v) d \mu_{1}(v) \\
& \leq\left(\int_{S M}-\hat{K}^{-}(v) d \mu_{1}(v)\right)^{1 / 2}(n-1) .
\end{aligned}
$$

Now integrating over the $(n-1)$ sphere at each point of $M$ first, and then over $M$, and remembering that $d \mu_{1}$ is normalized, leads to

$$
\frac{n-1}{V} \int_{M}\left(-K^{+}(x)\right)^{1 / 2} d V(x) \leq h_{\mu} \leq(n-1)\left(\frac{1}{V} \int_{M}-K^{-}(x) d V(x)\right)^{1 / 2}
$$

proving theorem 1 .

For the upper bound of theorem 2, we observe that the upper bound of lemma 2.1 holds (say in the case of $\operatorname{dim} M=2$ ) if

i.e.,

$$
c+c^{-1}(-\hat{K}) \geq 0
$$

$$
c^{2} \geq K(x)
$$

So in this case,

$$
\chi(v, \xi) \leq \frac{1}{2}\left(c+c^{-1} \varlimsup_{t \rightarrow \infty} \frac{1}{t} \int_{0}^{t}-K(u) d u\right) .
$$

Since $K$ may be non-negative, we do not necessarily have any Anosov property so we learn only that

$$
\chi(v) \leq c+c^{-1} \varlimsup_{t \rightarrow \infty} \frac{1}{t} \int_{0}^{t}-K(u) d u
$$

If

$$
\phi(v)=\lim _{t \rightarrow \infty} \frac{1}{t} \int_{0}^{t}-K(u) d u
$$

which exists almost everywhere by the Birkoff ergodic theorem, then we have

$$
\begin{aligned}
\chi(v) & \leq c+c^{-1} \phi(v) \\
\int_{S M} \chi(v) d \mu_{1}(v) & \leq c+c^{-1} \int_{S M} \chi(v) d \mu_{1}(v) \\
& =c+c^{-1} \int_{S M}-K(v) d \mu_{1}(v) \\
& =c+c^{-1} \frac{4 \pi(g-1)}{V}
\end{aligned}
$$

https://doi.org/10.1017/S0143385700001747 Published online by Cambridge University Press 
Therefore choosing

$$
c=\sqrt{\frac{4 \pi(g-1)}{V}}
$$

which by assumption makes (2.17) valid, shows that

$$
h_{\mu} \leq 2\left(\frac{4 \pi(g-1)}{V}\right)^{1 / 2}
$$

as needed.

We now turn to the proof of theorem 3 .

Let $\Gamma$ be the fundamental group of $M$, which acts as isometries on the universal covering $\tilde{M}$. $\Gamma$ is of course infinite. For $\gamma \in \Gamma$, let the operator $T_{\gamma}$ which acts on functions on $\dot{M}$ and in particular on $L^{2}(\tilde{M})$ be defined by

$$
T_{\gamma} f(x)=f(\gamma x) .
$$

Since $\gamma \in \Gamma$ is an isometry of $\tilde{M}$ it follows that

$$
T_{\gamma} \circ \tilde{\Delta}=\tilde{\Delta} \circ T_{\gamma} \text {. }
$$

With these remarks it is easy to see that the spectrum of $\tilde{\Delta}$ is essential - (for a definition of essential spectrum see Reed \& Simon [12]).

In fact, if $\lambda$ is an eigenvalue of $\tilde{\Delta}$, then it must be of infinite multiplicity. For suppose

$$
\tilde{\Delta} f=\lambda f \quad \text { with } f \in L^{2}(\tilde{M}) .
$$

It is clear that sufficiently large 'translates' of $f$ by motions $\gamma \in \Gamma$ will yield linearly independent functions in $L^{2}(\tilde{M})$ (since $f \in L^{2}$ ). On the other hand, it is clear from (2.18) that such a translate $f_{\gamma}$ is again an eigenfunction of $\tilde{\Delta}$ with eigenvalue $\lambda$.

The characterization of $h$ in terms of volumes mentioned in the beginning of $\S 2$ is as follows. Let $x$ be any point of $M$, and let $\tilde{x} \in \tilde{M}$ lie above $x$. Let

$$
V(\tilde{x}, R)=\text { Volume }(B(\tilde{x}, R))
$$

where $B(\tilde{x}, R)$ is a ball radius $R$ in $\dot{M}$ centred at $\tilde{x}$. Then independent of $x$

$$
h=\lim _{R \rightarrow \infty} \frac{\log V(\tilde{x}, R)}{R} .
$$

The bottom of the spectrum of $-\tilde{\Delta}, \beta$, may be described in the variational form

$$
\beta=\inf _{f \text { of compact support }} \frac{\int_{\dot{M}}|\nabla f|^{2} d V}{\int_{\dot{M}} f^{2} d V} .
$$

The estimate of theorem 3 will follow when we show that the 'Rayleigh quotient' can be made as close to $h^{2} / 4$ as we please by suitable choice of test functions.

Let $\varepsilon>0, \eta>0$ with $\varepsilon$ small and $\eta$ large. let $\phi$ be a positive $C^{\infty}$ function $\phi \in C_{0}^{\infty}(0, \infty)$ and $\phi \equiv 1$ on $[\xi, \eta]$. 
Take $x \in \tilde{M}$, and let $(r, \theta)$ be geodesic normal polar coordinates about $x$. Thus, $0<r<\infty, \theta \in s^{n-1}$; these give a global chart for $\tilde{M}$. The line elements takes the form

$$
d s^{2}=g_{i j} d x^{i} d x^{j}=d r^{2}+G_{i j} d \theta^{i} d \theta^{j}
$$

i.e.,

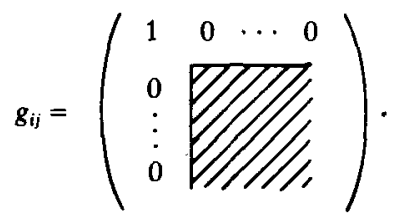

Let

$$
f(r, \theta)=\phi(r) \exp \left(-\left(h / 2+\varepsilon_{1}\right) r\right), \quad \varepsilon_{1}>0
$$

Then

$$
\begin{aligned}
Q(f) & =\frac{\int|\nabla f|^{2} d V}{\int f^{2} d V} \\
& =\frac{\int_{0}^{\infty} \int_{S^{n-1}}\left(\frac{\partial f}{\partial r}\right)^{2} \sqrt{G(r, \theta)} d \theta d r}{\int_{0}^{\infty} \int_{S^{n-1}} f^{2} \sqrt{G} d \theta d r} .
\end{aligned}
$$

Let

$$
S(r)=\int_{S^{n-1}} \sqrt{G(r, \theta)} d \theta
$$

which is the volume of the spherical shell radius $r$ about $x$. Since $S$ is increasing and

$$
V(x, R)=\int_{0}^{R} S(r) d r
$$

we see from (2.19) that

$$
S(r)=O\left(\exp h_{1} r\right) \quad \text { for any } h_{1}>h
$$

also

$$
S(r)=O\left(r^{n-1}\right) \quad \text { as } r \searrow 0
$$

Now

$$
Q(f)=\frac{\int_{0}^{\infty}\left(\frac{\partial f}{\partial r}\right)^{2} S(r) d r}{\int_{0}^{\infty} f^{2} S(r) d r}
$$

The estimates (2.20) allow us to let $\varepsilon \rightarrow 0$ and $\eta \rightarrow \infty$, and we have 


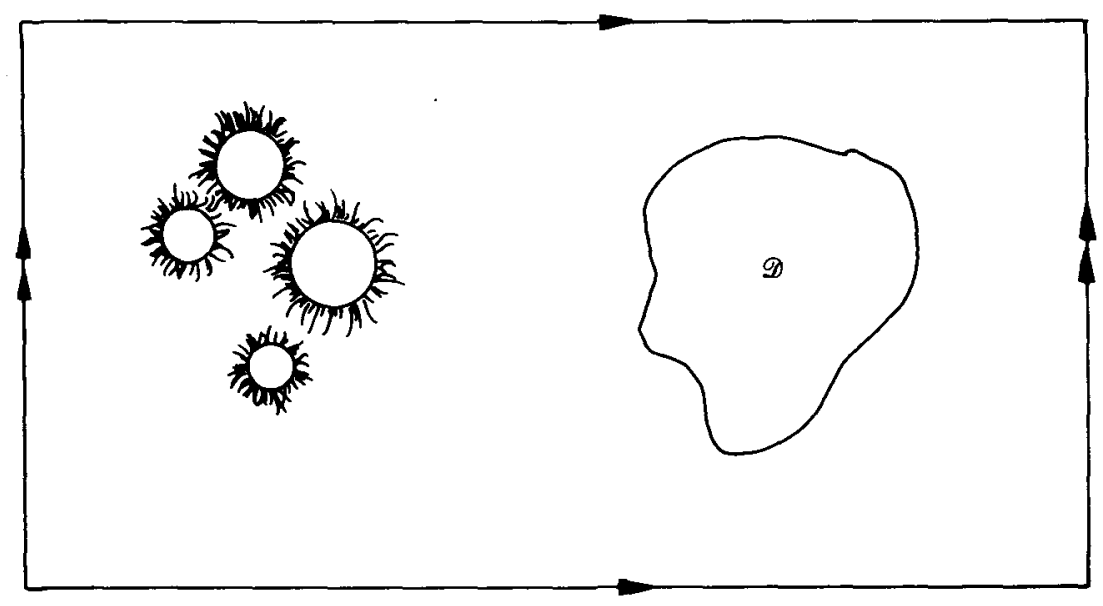

$$
\begin{aligned}
Q(f) & \rightarrow \frac{\int_{0}^{\infty} \exp -2\left(\frac{h}{2}+\varepsilon_{1}\right) r\left(\frac{h}{2}+\varepsilon_{1}\right)^{2} S(r) d r}{\int_{0}^{\infty} \exp -2\left(\frac{h}{2}+\varepsilon_{1}\right) r S(r) d r} \\
& =\left(\frac{h}{2}+\varepsilon_{1}\right)^{2}
\end{aligned}
$$

Since $\varepsilon_{1}$ is arbitrary,

$$
\beta \leq \frac{h^{2}}{4}
$$

In the case that $M$ and hence $\tilde{M}$ has constant negative curvature, say $K=-1$, then it is known, see for example Mckean [8], that

$$
\operatorname{spectrum}(-\tilde{\Delta})=\left[\left(\frac{n-1}{2}\right)^{2}, \infty\right]
$$

and is absolutely continuous. So that in this case the inequality of theorem 3 is sharp.

To show that the same inequality can be rather rough, consider a two-dimensional flat torus $M$ with $m$ small holes removed.

Now think of $M$ as having two sides and 'blow up' the picture slightly so that we have an $m+1$ holed torus of negative curvature with all the curvature concentrated on (or near) the rims of the holes. Let $\mathscr{D}$ be a simply connected sub-domain which is untouched.

Clearly, $\beta \leq \lambda_{1}(D)$ where $\lambda_{1}(D)$ is the smallest eigenvalue for the Dirichelet problem of $D$. One may remove the holes in such a fashion that the volume of $M$ remains essentially unchanged.

Using (1.3) it is evident that $h \rightarrow \infty$ with $m$, while $\beta$ remains bounded. 


\section{Section 3}

Question: What is the nature of the spectrum of $\tilde{\Delta}$ on $\check{M}$ ? One may guess in analogy with Bloch wave theory for a periodic potential (see Reed \& Simon [12]) that it consists of bands and is absolutely continuous.

Note. It has been pointed out to the author that A. Manning (1980) has obtained a similar lower bound for $h_{\mu}$, to that of corollary 1 .

This work was partially supported by NSF grant MCJ-7900813. Some of the results of this paper are part of the author's thesis, Stanford, 1980.

\section{REFERENCES}

[1] M. Berger. La Spectre d'une variete Riemmanienne. Springer Lecture Notes no. 194.

[2] R. Bowen. On axiom A diffeomorphisms. Conference Board of Math. Sciences. Regional Conf. Ser. in Math., Amer. Math. Soc.: Rhode Island, 35 (1978), 17-20.

[3] P. Eberlein. J. Diff. Geometry 8 (1973), 437-463.

[4] V. Guillemin \& J. Duistermaat. The spectrum of a positive elliptic operator and periodic bicharacteristics. Invent. Math. 29 (1975), 37-79.

[5] W. Goodwyn. Comparing topological entropy with measure entropy. Amer. J. of Math. 94 (1972), 336.

[6] A. Katok. Entropy and closed geodesics. Ergod. Th. \& Dynam. Sys. 2 (1982), 339.

[7] A. Manning. Topological entropy for geodesic flows, Annal Math. 110 (1979), 567-573.

[8] H. Mckean. An upper bound for the spectrum of $\Delta$ on a manifold of negative curvature, $J$. Diff. Geom. 4 (1970), 359.

[9] H. Mckean \& I. Singer. Curvature and eigenvalues of the Laplacian. J. Diff. Geom. 1 (1967), 43-67.

[10] G. Margulis. Applications of ergodic theory to the investigation of manifolds of negative curvature. Func. Anal. and Appl. 3 (1969), 335-336.

[11] Ya. Pesin. Russian Math. Surveys 32-4 (1977), 55-114.

[12] M. Reed \& B. Simon. Meth. Math. Phys. vol. 4, Academic Press: New York, 1978, p. 279.

[13] Ja. Sinai. Introduction to Ergodic Theory. Princeton Univ. Press: Princeton, 1976. p. 119.

[14] Ja. Sinai. The asymptotic behaviour of the number of closed geodesics. A.M.S. Transl. 73 (1968), $227-250$. 